\title{
A static formulation for topology optimization with control action
}

\author{
$\underline{\text { Lucas dos Santos Fernandez }}^{*} \quad$ Alexandre Molter Fabio Silva Botelho \\ Department of Mathematics and Statistics, UFPel, \\ Campus Capão do Leão, 96010-900, RS \\ E-mail: lucassfernandez@gmail.com, alexandre.molter@ufpel.edu.br, fabio.silva.botelho@gmail.com
}

\begin{abstract}
$\underline{\text { ABSTRACT }}$ element was considered relating the Finite Element Method (FEM). depends non-linearly on $\rho_{2}$. The compliance optimization can be formulated as

$$
\begin{array}{cc}
\min _{\rho_{1}, \rho_{2}, u} & l(u), \\
\text { s.t. } & a\left(\rho_{1}, \rho_{2}, u, v\right)-l(v) \\
& \int_{\Omega} \rho_{1} d \Omega=\eta_{0} V, \\
& 0<\rho_{1_{\text {min }}} \leq \rho_{1} \leq 1, \text { in } \Omega, \\
& \int_{\Omega} \rho_{2} d \Omega \leq \eta_{1} V, \\
& 0<\rho_{2_{\text {min }}} \leq \rho_{2} \leq 1, \text { in } \Omega,
\end{array}
$$$$
\text { s.t. } \quad a\left(\rho_{1}, \rho_{2}, u, v\right)-l(v) \quad=0 \quad \forall v,
$$

In this work we consider the classical minimum compliance topology optimization in linear elasticity. In our formulation we consider a control functional that represents control forces acting on a structural piece intending to minimize a specific cost functional. The cost functional in question comprises the work of applied body and traction forces. The optimization procedure is performed under suitable constraints for external forces, control energy, volume restriction and a set of project variables. We highlight the variational problem addressed may be summarized as the search for the optimal density distributions of material and control forces which minimize the inner structural work. The topology optimization in this work uses Solid Isotropic Material with Penalization approach (SIMP) [3, 4], based on the concept of optimizing the material distribution, through a density distribution, while the control force is inserted in steady-state form. In order to model the structure, namely a cantilever beam, a bilinear iso-parametric

At this point we start to describe the primal variational formulation. Let $\Omega$ be a Lipschitzian domain with a boundary $\Gamma$. The boundary $\Gamma$ is divided in two parts, $\Gamma_{t}$ and $\Gamma_{u}$, such that measure $e_{\Omega}\left(\Gamma_{t} \cap \Gamma_{u}\right)=$ 0 and $\Gamma_{t} \cup \Gamma_{u}=\Gamma$. We define pseudo-density functions $\rho_{1}(x)$ and $\rho_{2}(x)$ on $\Omega$ such that the fourth order elasticity tensor $E\left(\rho_{1}\right)$ depends, through a penalty parameter, non-linearly on $\rho_{1}(x)$, whereas $h\left(\rho_{2}\right)$

where $\eta_{0}$ and $\eta_{1}$ are the prescribed volume fractions, $\rho_{1_{\text {min }}}$ and $\rho_{2_{\text {min }}}$ are the minimum relative densities and

$$
l(u)=\int_{\Omega} f \cdot u d \Omega+\int_{\Gamma_{t}} t \cdot u d \Gamma,
$$

is the work of the external forces, $t$ is the boundary (surface) force, $u$ is the displacement vector and $f$ is the body force, which can also contain the boundary and point loads with singularity functions. The term

$$
a\left(\rho_{1}, \rho_{2}, u, v\right)=\int_{\Omega} \varepsilon(u): E\left(\rho_{1}\right): \varepsilon(v) d \Omega+\int_{\Omega} r h\left(\rho_{2}\right) u \cdot v d \Omega,
$$

is the virtual work of the stresses, where $\varepsilon$ is the infinitesimal displacement-deformation (symmetrized gradient) operator, that is, $\varepsilon(u)=\frac{1}{2}\left(\nabla u+(\nabla u)^{T}\right) \cdot v \in U$ has the role of a Lagrange multiplier, that is, $v=u_{0}$ in $\Gamma_{u}$. The term $E\left(\rho_{1}\right)=\rho_{1}^{p} E^{0}$ is the fourth order positive definite elastic constitutive tensor.

\footnotetext{
${ }^{*}$ Grateful for CAPES financial support.
} 
The term $h\left(\rho_{2}\right)=\rho_{2}^{p} h^{0}$ is a unity localization function and $r$ a scalar magnitude. In both $E\left(\rho_{1}\right)$ and $h\left(\rho_{2}\right), p$ is an arbitrary penalty exponent whose value is 3 [4].

The continuum optimization problem (1) was discretized in finite elements. The shape functions relating the bilinear iso-parametric element were chosen as shown in [1]. Thus, the discrete objective function may be write in matrix form as $J\left(\rho_{1}, \rho_{2}, u\right)=\sum_{e=1}^{n}\left(\rho_{1_{e}}\right)^{p} \mathbf{u}_{e}^{T} \mathbf{k}_{e} \mathbf{u}_{e}+\sum_{e=1}^{n}\left(\rho_{2_{e}}\right)^{p} r \mathbf{u}_{e}^{T} \mathbf{h}_{e} \mathbf{u}_{e}$, where $\mathbf{u}_{e}$ is elemental deflection, $\mathbf{k}_{e}$ is the elemental stiffness matrix, $e$ is the elemental index. The discretization was also extended to constraints.

The sensitivities are defined as the derivatives of the objective function and constraints with respect to the design variables. In this formulation, the objective function and the constraints sensitivity are derived through the Lagrangian $\mathcal{L}$, where

$$
\begin{aligned}
& \mathcal{L}=l(u)-\left\{a\left(\rho_{1}, \rho_{2}, u, v\right)-l(v)\right\}+\Lambda_{1}\left(\int_{\Omega} \rho_{1} d \Omega-\eta_{0} V\right)+\int_{\Omega} \lambda_{1}\left(\rho_{1}-1\right) d \Omega \\
& +\int_{\Omega} \lambda_{2}\left(\rho_{1_{\text {min }}}-\rho_{1}\right) d \Omega+\Lambda_{2}\left(\int_{\Omega} \rho_{2} d \Omega-\eta_{1} V\right)+\int_{\Omega} \lambda_{3}\left(\rho_{2}-1\right) d \Omega+\int_{\Omega} \lambda_{4}\left(\rho_{2_{\text {min }}}-\rho_{2}\right) d \Omega,
\end{aligned}
$$

where the Lagrange multipliers $\Lambda_{1}, \Lambda_{2} \in \mathbb{R}, \lambda_{1}, \lambda_{2}, \lambda_{3}, \lambda_{4}$ are scalar functions and the variations of $\mathcal{L}$ are calculated with respect to $u, v, \rho_{1}$ and $\rho_{2}$.

In the numerical simulations we considered the following physics values: $E=210 \times 10^{9}=$ $210 G P a, \nu=0.3, \rho_{1_{\text {min }}}=0.001, \rho_{2_{\text {min }}}=0.001, \eta_{0}=0.5, \eta_{1}=0.1$ and $F=-1 \times 10^{6} \mathrm{~N}$. The finite element mesh considered comprises about $40 \times 16$ elements.

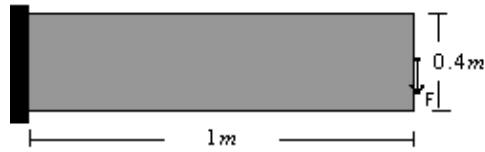

(a)

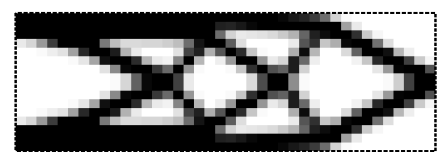

(b)

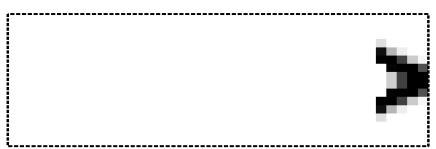

(c)

The Figure (a) shows the cantilever beam with no loads. The topology of the optimal structure is shown in Figure (b). Figure (c) refers to the optimal control variable distribution. One can observe that there is presence of material where the displacement is larger, since the control energy is dependent on displacements.

Although the variational formulations have not been presented, they were developed in the full work. From our results we may conclude the structure changes when the control energy is included. Despite not being directly applicable to the real structural pieces, due to manufacturing limitations, the study is interesting to show where the optimal controllers are located in order to minimize the inner structural work. The results are consistent with the problem physics.

Keywords: Variational formulation, Topology optimization, Control

\section{Referências}

[1] E. B. Becker, G.F. Carey, J. T. Oden, "Finite Elements: an Introduction", Prentice-Hall, v. I, New Jersey, 1981

[2] M. P. Bendsøe, O. Sigmund, "Topology Optimization - Theory, Methods and Applications", Springer, New York, 2003

[3] J.S. Ou, N. Kikuchi, Optimal design of controlled structures, Structural Optimization, 11 (1996) $19-28$

[4] O. Sigmund, A 99 line topology optimization code written in Matlab, Structural Multidisciplinary Optimization, 21 (2001) 120-127 